\title{
Fixed Point of a Countable Family of Uniformly Totally Quasi- $\phi$-Asymptotically Nonexpansive Multi-Valued Mappings in Reflexive Banach Spaces with Applications
}

\author{
Yi Li \\ School of Science, Southwest University of Science and Technology, Mianyang, China \\ Email: liyi@swust.edu.cn
}

Received April 23, 2013; revised May 23, 2013; accepted June 1, 2013

Copyright (C) 2013 Yi Li. This is an open access article distributed under the Creative Commons Attribution License, which permits unrestricted use, distribution, and reproduction in any medium, provided the original work is properly cited.

\begin{abstract}
The purpose of this article is to discuss a modified Halpern-type iteration algorithm for a countable family of uniformly totally quasi- $\phi$-asymptotically nonexpansive multi-valued mappings and establish some strong convergence theorems under certain conditions. We utilize the theorems to study a modified Halpern-type iterative algorithm for a system of equilibrium problems. The results improve and extend the corresponding results of Chang et al. (Applied Mathematics and Computation, 218, 6489-6497).
\end{abstract}

Keywords: Multi-Valued Mapping; Totally Quasi- $\phi$-Asymptotically Nonexpansive; Countable Family of Uniformly Totally Quasi- $\phi$-Asymptotically Nonexpansive Multi-Valued Mappings; Firmly Convergence

\section{Introduction}

Throughout this paper, we denote the strong convergence and weak convergence of the sequence $\left\{x_{n}\right\}$ by $x_{n} \rightarrow x$ and $x_{n} \rightarrow x$, respectively. We denote by $N$ and $R$ the sets of positive integers and real numbers, respectively. Let $D$ be a nonempty closed subset of a real Banach space $X$. A mapping $T: D \rightarrow D$ is said to be nonexpansive if $\|T x-T y\| \leq\|x-y\|$, for all $x, y \in D$. Let $N(D)$ and $C B(D)$ denote the family of nonempty subsets and nonempty bounded closed subsets of $D$, respectively.

Let $X$ be a real Banach space with dual $X^{*}$. We denote by $J$ the normalized duality mapping from $X$ to $2^{X^{*}}$ which is defined by

$J(x)=\left\{x^{*} \in X^{*}:\left\langle x, x^{*}\right\rangle=\|x\|^{2}=\left\|x^{*}\right\|^{2}\right\}$, where $x \in X$ and $\langle\cdot, \cdot\rangle$ denotes the generalized duality pairing. The Hausdorff metric on $C B(D)$ is defined by

$H\left(A_{1}, A_{2}\right)=\max \left\{\sup _{x \in A_{1}} d\left(x, A_{2}\right), \sup _{y \in A_{2}} d\left(y, A_{1}\right)\right\}$, for

$A_{1}, A_{2} \in C B(D)$, where $d\left(x, A_{2}\right)=\inf \left\{\|x-y\|, y \in A_{2}\right\}$. The multi-valued mapping $T: D \rightarrow C B(D)$ is called nonexpansive if $H(T x, T y) \leq\|x-y\|$ for all $x, y \in D$. An element $p \in D$ is called a fixed point of
$T: D \rightarrow C B(D)$ if $p \in T(p)$. The set of fixed points of $T$ is represented by $F(T)$. In the sequel, denote $S(X)=\{x \in X:\|x\|=1\}$. A Banach space $X$ is said to be strictly convex if $\left\|\frac{x+y}{2}\right\| \leq 1$ for all $x, y \in S(X)$ and $x \neq y$. A Banach space is said to be uniformly convex if $\lim _{n \rightarrow \infty}\left\|x_{n}-y_{n}\right\|=0$ for any two sequences $\left\{x_{n}\right\},\left\{y_{n}\right\} \subset S(X)$ and $\lim _{n \rightarrow \infty} \frac{\left\|x_{n}+y_{n}\right\|}{2}=0$. The norm of Banach space $X$ is said to be Gâteaux differentiable if for each $x, y \in S(X)$, the limit

$$
\lim _{t \rightarrow 0} \frac{\|x+t y\|-\|x\|}{t}
$$

exists. In this case, $X$ is said to be smooth. The norm of Banach space $X$ is said to be Fréchet differentiable, if for each $x \in S(X)$, the limit (1.1) is attained uniformly for $y \in S(x)$ and the norm is uniformly Fréchet differentiable if the limit (1.1) is attained uniformly for $x, y \in S(X)$. In this case, $X$ is said to be uniformly smooth.

The following basic properties for Banach space $X$ and for the normalized duality mapping $J$ can be found in Cioranescu [1].

(1) $X\left(X^{*}\right.$, resp. $)$ is uniformly convex if and only if 
$X^{*}(X$, resp. $)$ is uniformly smooth.

(2) If $X$ is smooth, then $J$ is single-valued and norm-to-weak ${ }^{*}$ continuous.

(3) If $X$ is reflexive, then $J$ is onto.

(4) If $X$ is strictly convex, then $J x \cap J y \neq \Phi$ for all $x, y \in X$.

(5) If $X$ has a Fréchet differentiable norm, then $J$ is norm-to-norm continuous.

(6) If $X$ is uniformly smooth, then $J$ is uniformly norm-to-norm continuous on each bounded subset of $X$.

(7) Each uniformly convex Banach space $X$ has the Kadec-Klee property, i.e., for any sequence $\left\{x_{n}\right\} \subset X$, if $x_{n} \rightarrow x \in X$ and $\left\|x_{n}\right\| \rightarrow\|x\|$, then $x_{n} \rightarrow x \in X$.

In 1953, Mann [2] introduced the following iterative sequence $\left\{x_{n}\right\}$,

$$
x_{n+1}=\alpha_{n} x_{n}+\left(1-\alpha_{n}\right) T x_{n},
$$

where the initial guess $x_{1} \in D$ is arbitrary and $\left\{\alpha_{n}\right\}$ is a real sequence in $[0,1]$. It is known that under appropriate settings the sequence $\left\{x_{n}\right\}$ converges weakly to a fixed point of $T$. However, even in a Hilbert space, Mann iteration may fail to converge strongly [3]. Some attempts to construct iteration method guaranteeing the strong convergence have been made. For example, Halpern [4] proposed the following so-called Halpern iteration,

$$
x_{n+1}=\alpha_{n} u+\left(1-\alpha_{n}\right) T x_{n},
$$

where $u, x_{1} \in D$ are arbitrary given and $\left\{\alpha_{n}\right\}$ is a real sequence in $[0,1]$. Another approach was proposed by Nakajo and Takahashi [5]. They generated a sequence as follows,

$$
\left\{\begin{array}{l}
x_{1} \in X \text { is arbitrary; } \\
y_{n}=\alpha_{n} u+\left(1-\alpha_{n}\right) T x_{n} \\
C_{n}=\left\{z \in D:\left\|y_{n}-z\right\| \leq\left\|x_{n}-z\right\|\right\} \\
Q_{n}=\left\{z \in D:\left\langle x_{n}-z, x_{1}-x_{n}\right\rangle \geq 0\right\} \\
x_{n+1}=P_{C_{n} \cap Q_{n}} x_{1}(n=1,2, \cdots)
\end{array}\right.
$$

where $\left\{\alpha_{n}\right\}$ is a real sequence in $[0,1]$ and $P_{K}$ denotes the metric projection from a Hilbert space $H$ onto a closed convex subset $K$ of $H$. It should be noted here that the iteration above works only in Hilbert space setting. To extend this iteration to a Banach space, the concept of relatively nonexpansive mappings and quasi- $\phi$-nonexpansive mappings are introduced by Aoyama et al. [6], Chang et al. [7,8], Chidume et al. [9], Matsushita et al. [10-12], Qin et al. [13], Song et al. [14], Wang et al. [15] and others.

Inspired by the work of Matsushita and Takahashi, in this paper, we introduce modifying Halpern-Mann iterations sequence for finding a fixed point of a countable family of uniformly totally quasi- $\phi$-asymptotically nonexpansive multi-valued mappings in reflexive Banach spaces $T_{i}: D \rightarrow D(i=1,2,3, \cdots)$ and some strong convergence theorems are proved. The results presented in the paper improve and extend the corresponding results in [7].

\section{Preliminaries}

In the sequel, we assume that $X$ is a smooth, strictly convex, and reflexive Banach space and $D$ is a nonempty closed convex subset of $X$. In the sequel, we always use $\phi: X \times X \rightarrow R^{+}$to denote the Lyapunov bifunction defined by

$$
\phi(x, y)=\|x\|^{2}-2\langle x, J y\rangle+\|y\|^{2}, x, y \in X .
$$

It is obvious from the definition of the function $\phi$ that

$$
\begin{aligned}
& (\|x\|-\|y\|)^{2} \leq \phi(x, y) \leq(\|x\|+\|y\|)^{2}, \\
& \phi(y, x)=\phi(y, z)+\phi(z, x) \\
& +2\langle z-y, J x-J z\rangle, \quad x, y, z \in X,
\end{aligned}
$$

and

$$
\begin{aligned}
& \phi\left(x, J^{-1}(\alpha J y+(1-\alpha) J z)\right) \\
& \leq \alpha \phi(x, y)+(1-\alpha) \phi(x, z)
\end{aligned}
$$

for all $\alpha \in[0,1]$ and $x, y, z \in X$.

Following Alber [16], the generalized projection $\Pi_{D}: X \rightarrow D$ is defined by

$$
\Pi_{D}(x)=\arg y \in D \inf \phi(y, x), \forall x \in X .
$$

Many problems in nonlinear analysis can be reformulated as a problem of finding a fixed point of a nonexpansive mapping.

Remark 2.1 (see [17]) Let $\Pi_{D}$ be the generalized projection from a smooth, reflexive and strictly convex Banach space $X$ onto a nonempty closed convex subset $D$ of $X$, then $\Pi_{D}$ is a closed and quasi- $\phi$-nonexpansive from $X$ onto $D$.

Lemma 2.1 (see [16]) Let $X$ be a smooth, strictly convex and reflexive Banach space and $D$ be a nonempty closed convex subset of $X$. Then the following conclusions hold,

(a) $\phi(x, y)=0$ if and only if $x=y$.

(b) $\phi\left(x, \Pi_{D} y\right)+\phi\left(\Pi_{D} y, y\right) \leq \phi(x, y), \forall x, y \in D$.

(c) If $x \in X$ and $z \in D$, then $z=\Pi_{D} x$ if and only if $\langle z-y, J x-J z\rangle \geq 0, \forall y \in D$.

Lemma 2.2 (see [7]) Let $X$ be a real uniformly smooth and strictly convex Banach space with KadecKlee property, and $D$ be a nonempty closed convex subset of $X$. Let $\left\{x_{n}\right\}$ and $\left\{y_{n}\right\}$ be two sequences in $D$ such that $x_{n} \rightarrow p$ and $\phi\left(x_{n}, y_{n}\right) \rightarrow 0$ where $\phi$ 
is the function defined by (1.2), then $y_{n} \rightarrow p$.

Definition 2.1 A point $p \in D$ is said to be an asymptotic fixed point of multi-valued mapping

$T: D \rightarrow C B(D)$, if there exists a sequence $\left\{x_{n}\right\} \subset D$ such that $x_{n} \rightarrow x \in X$ and $d\left(x_{n}, T\left(x_{n}\right)\right) \rightarrow 0$. Denote the set of all asymptotic fixed points of $T$ by $\hat{F}(T)$.

Definition 2.2

(1) A multi-valued mapping $T: D \rightarrow C B(D)$ is said to be relatively nonexpansive, if $F(T) \neq \Phi$,

$\hat{F}(T)=F(T)$, and $\phi(p, z) \leq \phi(p, x), \forall x \in D$, $p \in F(T), z \in T(x)$.

(2) A multi-valued mapping $T: D \rightarrow C B(D)$ is said to be closed, if for any sequence $\left\{x_{n}\right\} \subset D$ with $x_{n} \rightarrow x \in X$ and $d\left(y, T\left(x_{n}\right)\right) \rightarrow 0$, then $d(y, T(x))=0$.

Remark 2.2 If $H$ is a real Hilbert space, then $\phi(x, y)=\|x-y\|^{2}$ and $\Pi_{D}$ is the metric projection $P_{D}$ of $H$ onto $D$.

Next, We present an example of relatively nonexpansive multi-valued mapping.

Example 2.1 (see [18]) Let $X$ be a smooth, strictly convex and reflexive Banach space, $D$ be a nonempty closed and convex subset of $X$ and $f: D \times D \rightarrow R$ be a bifunction satisfying the conditions:

(A1) $f(x, x)=0, \forall x \in D$;

(A2) $f(x, y)+f(y, x) \leq 0, \forall x, y \in D$;

(A3) for each $x, y, z \in D$,

$$
\lim _{t \rightarrow 0} f(t z+(1-t) x, y) \leq f(x, y) ;
$$

(A4) for each given $x \in D$, the function $y \mapsto f(x, y)$ is convex and lower semicontinuous.

The "so-called" equilibrium problem for $f$ is to find a $x^{*} \in D$ such that $f\left(x^{*}, y\right) \geq 0, \forall y \in D$. The set of its solutions is denoted by EP( $f)$.

Let $r>0, x \in D$ and define a multi-valued mapping $T_{r}: D \rightarrow N(D)$ as follows,

$$
\begin{aligned}
& T_{r}(x) \\
& =\left\{z \in D, f(z, y)+\frac{1}{r}\langle y-z, J z-J x\rangle \geq 0, \forall y \in D\right\}, \\
& \forall x \in D,
\end{aligned}
$$

then (1) $T_{r}$ is single-valued, and so $\{z\}=T_{r}(x)$; (2) $T_{r}$ is a relatively nonexpansive mapping, therefore, it is a closed quasi- $\phi$-nonexpansive mapping; (3)

$$
F\left(T_{r}\right)=E P(f) \text {. }
$$

\section{Definition 2.3}

(1) A multi-valued mapping $T: D \rightarrow C B(D)$ is said to be quasi- $\phi$-nonexpansive, if $F(T) \neq \Phi$, and $\phi(p, z) \leq \phi(p, x), \forall x \in D, p \in F(T), z \in T x$.

(2) A multi-valued mapping $T: D \rightarrow C B(D)$ is said to be quasi- $\phi$-asymptotically nonexpansive, if $F(T) \neq \Phi$ and there exists a real sequence $k_{n} \subset[1,+\infty), k_{n} \rightarrow 1$ such that

$$
\begin{aligned}
& \phi\left(p, z_{n}\right) \leq k_{n} \phi(p, x), \forall x \in D, \\
& p \in F(T), z_{n} \in T^{n} x ;
\end{aligned}
$$

(3) A multi-valued mapping $T: D \rightarrow C B(D)$ is said to be totally quasi- $\phi$-asymptotically nonexpansive, if $F(T) \neq \Phi$ and there exist nonnegative real sequences $\left\{v_{n}\right\},\left\{\mu_{n}\right\}$, with $v_{n}, \mu_{n} \rightarrow 0 \quad$ (as $\left.n \rightarrow \infty\right)$ and a strictly increasing continuous function $\zeta: R^{+} \rightarrow R^{+}$with $\zeta(0)=0$ such that

$$
\begin{gathered}
\phi\left(p, z_{n}\right) \leq \phi(p, x)+v_{n} \zeta[\phi(p, x)]+\mu_{n}, \\
\forall x \in D, \forall n \geq 1, p \in F(T), z_{n} \in T^{n} x .
\end{gathered}
$$

Remark 2.3 From the definitions, it is obvious that a relatively nonexpansive multi-valued mapping is a quasi$\phi$-nonexpansive multi-valued mapping, and a quasi- $\phi$ nonexpansive multi-valued mapping is a quasi- $\phi$-asymptotically nonexpansive multi-valued mapping, and a quasi- $\phi$-asymptotically nonexpansive multi-valued mapping is a total quasi- $\phi$-asymptotically nonexpansive multi-valued mapping, but the converse is not true.

Lemma 2.3 Let $X$ and $D$ be as in Lemma 2.2. $T: D \rightarrow C B(D)$ be a closed and totally quasi- $\phi$-asymptotically nonexpansive multi-valued mapping with nonnegative real sequences $\left\{v_{n}\right\},\left\{\mu_{n}\right\}$ and a strictly increasing continuous function $\zeta: R^{+} \rightarrow R^{+}$with $\zeta(0)=0 \quad$ if $v_{n}, \mu_{n} \rightarrow 0 \quad$ (as $n \rightarrow \infty$ ) and $\mu_{1}=0$, then $F(T)$ is a closed and convex subset of $D$.

Proof. Let $\left\{x_{n}\right\}$ be a sequence in $F(T)$, such that $x_{n} \rightarrow x^{*}$. Since $T$ is totally quasi- $\phi$-asymptotically nonexpansive multi-valued mapping, we have

$$
\phi\left(x_{n}, z\right) \leq \phi\left(x_{n}, x^{*}\right)+v_{1} \zeta\left[\phi\left(x_{n}, x^{*}\right)\right]
$$

for all $z \in T x^{*}$ and for all $n \in N$. Therefore,

$$
\begin{aligned}
\phi\left(x^{*}, z\right) & =\lim _{n \rightarrow \infty} \phi\left(x_{n}, z\right) \\
& \leq \lim _{n \rightarrow \infty}\left\{\phi\left(x_{n}, x^{*}\right)+v_{1} \zeta\left[\phi\left(x_{n}, x^{*}\right)\right]\right\} \\
& =\phi\left(x^{*}, x^{*}\right)=0 .
\end{aligned}
$$

By Lemma 2.1(a), we obtain $z=x^{*}$. Hence, $T x^{*}=\left\{x^{*}\right\}$. So, we have $x^{*} \in F(T)$. This implies $F(T)$ is closed.

Let $p, q \in F(T)$ and $t \in(0,1)$, and put $w=t p+(1-t) q$. Next we prove that $w \in F(T)$. Indeed, in view of the definition of $\phi$, letting $z_{n} \in T^{n} w$, we have

$$
\begin{aligned}
\phi\left(w, z_{n}\right)= & \|w\|^{2}-2\left\langle w, J z_{n}\right\rangle+\left\|z_{n}\right\|^{2} \\
= & \|w\|^{2}-2\left\langle t p+(1-t) q, J z_{n}\right\rangle+\left\|z_{n}\right\|^{2} \\
= & \|w\|^{2}+t \phi\left(p, z_{n}\right)+(1-t) \phi\left(q, z_{n}\right) \\
& -t\|p\|^{2}-(1-t)\|q\|^{2} .
\end{aligned}
$$


Since

$$
\begin{aligned}
t \phi & \left(p, z_{n}\right)+(1-t) \phi\left(q, z_{n}\right) \\
\leq & t\left[\phi(p, w)+v_{n} \zeta[\phi(p, w)]+\mu_{n}\right] \\
& +(1-t)\left[\phi(q, w)+v_{n} \zeta[\phi(q, w)]+\mu_{n}\right] \\
= & t\left\{\|p\|^{2}-2\langle p, J w\rangle+\|w\|^{2}+v_{n} \zeta[\phi(p, w)]+\mu_{n}\right\} \\
& +(1-t)\left\{\|q\|^{2}-2\langle q, J w\rangle+\|w\|^{2}+v_{n} \zeta[\phi(q, w)]+\mu_{n}\right\} \\
= & t\|p\|^{2}+(1-t)\|q\|^{2}-\|w\|^{2}+t v_{n} \zeta[\phi(p, w)] \\
& +(1-t) v_{n} \zeta[\phi(q, w)]+\mu_{n} .
\end{aligned}
$$
have

Substituting (2.8) into (2.9) and simplifying it, we

$$
\begin{aligned}
\phi\left(w, z_{n}\right) \leq & t v_{n} \zeta[\phi(p, w)]+(1-t) v_{n} \zeta[\phi(q, w)] \\
& +\mu_{n} \rightarrow 0 . \quad(\text { as } n \rightarrow \infty)
\end{aligned}
$$

By Lemma 2.2, we have $z_{n} \rightarrow w$. This implies that $z_{n+1}\left(\in T T^{n} w\right) \rightarrow w$. Since $T$ is closed, we have

$T w=\{w\}$, i.e., $w \in F(T)$. This completes the proof of Lemma 2.3.

Definition 2.4 A mapping $T: D \rightarrow C B(D)$ is said to be uniformly $L$-Lipschitz continuous, if there exists a constant $L>0$ such that $\left\|x_{n}-y_{n}\right\| \leq L\|x-y\|$, where $x, y \in D, x_{n} \in T^{n} x, y_{n} \in T^{n} y$.

Definition 2.5

(1) A countable family of mappings $\left\{T_{i}\right\}: D \rightarrow D$ is said to be uniformly quasi- $\phi$-nonexpansive, if

$$
\begin{aligned}
F:=\bigcap_{i=1}^{\infty} F\left(T_{i}\right) & \neq \varnothing, \text { and } \\
& \phi(p, z) \leq \phi(p, x), \forall x \in D, p \in F, z \in T_{i} x .
\end{aligned}
$$

(2) A countable family of mappings $\left\{T_{i}\right\}: D \rightarrow D$ is said to be uniformly quasi- $\phi$-asymptotically nonexpansive, if $F:=\bigcap_{i=1}^{\infty} F\left(T_{i}\right) \neq \varnothing$, and there exists a real sequence $k_{n} \subset[1,+\infty), k_{n} \rightarrow 1$ such that,

$$
\phi\left(p, z_{n}\right) \leq k_{n} \phi(p, x), \forall x \in D, p \in F, z_{n} \in T_{i}^{n} x .
$$

(3) A countable family of mappings $\left\{T_{i}\right\}: D \rightarrow D$ is said to be totally uniformly quasi- $\phi$-asymptotically nonexpansive multi-valued, if $F:=\bigcap_{i=1}^{\infty} F\left(T_{i}\right) \neq \varnothing$ and there exists nonnegative real sequences $\left\{v_{n}\right\},\left\{\mu_{n}\right\}$ with $v_{n} \rightarrow 0, \mu_{n} \rightarrow 0$ (as $n \rightarrow \infty$ ) and a strictly increasing and continuous function $\zeta: \mathfrak{R}^{+} \rightarrow \mathfrak{R}^{+}$with $\zeta(0)=0$ such that

$$
\begin{gathered}
\phi\left(p, z_{n}\right) \leq \phi(p, x)+v_{n} \zeta[\phi(p, x)]+\mu_{n}, \\
\forall x \in D, \forall n \geq 1, p \in F, z_{n} \in T_{i}^{n} x .
\end{gathered}
$$

Remark 2.4 From the definitions, it is obvious that a countable family of uniformly quasi- $\phi$-nonexpansive multi-valued mappings is a countable family of uniformly quasi- $\phi$-asymptotically nonexpansive multi-valued mappings, and a countable family of uniformly quasi- $\phi$-asymptotically nonexpansive multi-valued mappings is a countable family of totally uniformly quasi- $\phi$-asymptotically multi-valued mappings, but the converse is not true.

\section{Main Results}

Theorem 3.1 Let $X$ be a real uniformly smooth and strictly convex Banach space with Kadec-Klee property, $D$ be a nonempty closed convex subset of $X$,

$T_{i}: D \rightarrow D, i=1,2,3, \cdots$ be a closed and uniformly $L_{i}-$ Lipschitz continuous and a countable family of uniformly totally quasi- $\phi$-asymptotically nonexpansive multi-valued mappings with nonnegative real sequences $\left\{v_{n}\right\}$, $\left\{\mu_{n}\right\}, v_{n}, \mu_{n} \rightarrow 0$ (as $\left.n \rightarrow \infty\right)$ and a strictly increasing continuous function $\zeta: R^{+} \rightarrow R^{+}$with

$\zeta(0)=0$ satisfying condition (2.11). Let $\left\{\alpha_{n}\right\}$ be a sequence in $[0,1]$ such that $\alpha_{n} \rightarrow 0$. If $\left\{x_{n}\right\}$ is the sequence generated by

$$
\left\{\begin{array}{l}
x_{1} \in X \text { is arbitrary; } D_{1}=D \\
y_{n, i}=J^{-1}\left[\alpha_{n} J x_{1}+\left(1-\alpha_{n}\right) J z_{n}\right], \quad z_{n} \in T_{i}^{n} x_{n}, i \geq 1 \\
D_{n+1}=\left\{z \in D_{n}: \sup _{i \geq 1} \phi\left(z, y_{n, i}\right)\right. \\
\left.\quad \leq \alpha_{n} \phi\left(z, x_{1}\right)+\left(1-\alpha_{n}\right) \phi\left(z, x_{n}\right)+\xi_{n}\right\} \\
x_{n+1}=\prod_{D_{n+1}} x_{1}(n=1,2, \cdots)
\end{array}\right.
$$

where $\xi_{n}=v_{n} \sup _{p \in F} \zeta\left[\phi\left(p, x_{n}\right)\right]+\mu_{n}, F\left(T_{i}\right)$ is the fixed point set of $T_{i}$, and $\Pi_{D_{n+1}}$ is the generalized projection of $X$ onto $D_{n+1}$.

$$
\text { If } F:=\bigcap_{i=1}^{\infty} F\left(T_{i}\right) \neq \varnothing \text { and } F \text { is bounded and }
$$

$\mu_{1}=0$, then $\lim _{n \rightarrow \infty} x_{n}=\Pi_{F} x_{1}$.

Proof. (I) First, we prove that $F$ and $D_{n} \quad(n \geq 1)$ are closed and convex subsets in $D$. In fact, it follows from Lemma 2.3 that $F\left(T_{i}\right)(i \geq 1)$ is a closed and convex subsets in $D$. Therefore $F$ is closed and convex subsets in $D$. Again by the assumption, $D_{1}=D$ is closed and convex. Suppose that $D_{n}$ is closed and convex for some $n \geq 1$. In view of the definition of $\phi$, we have

$$
\begin{aligned}
& D_{n+1}=\left\{z \in D_{n}: \sup _{i \geq 1} \phi\left(z, y_{n, i}\right) \leq \alpha_{n} \phi\left(z, x_{1}\right)\right. \\
&\left.+\left(1-\alpha_{n}\right) \phi\left(z, x_{n}\right)+\xi_{n}\right\} \\
&=\bigcap_{i \geq 1}\left\{z \in D: \phi\left(z, y_{n, i}\right) \leq \alpha_{n} \phi\left(z, x_{1}\right)\right. \\
&\left.\quad\left(1-\alpha_{n}\right) \phi\left(z, x_{n}\right)+\xi_{n}\right\} \cap D_{n} \\
&=\bigcap_{i \geq 1}\left\{z \in D: 2 \alpha_{n}\left\langle z, J x_{1}\right\rangle+2\left(1-\alpha_{n}\right)\left\langle z, J x_{n}\right\rangle-2\left\langle z, J y_{n, i}\right\rangle\right. \\
& \leq\left.\alpha_{n}\left\|x_{1}\right\|^{2}+\left(1-\alpha_{n}\right)\left\|x_{n}\right\|^{2}-\left\|y_{n, i}\right\|^{2}\right\} \cap D_{n} .
\end{aligned}
$$


This shows that $D_{n+1}$ is closed and convex. The conclusions are proved.

(II) Next, we prove that $F \subset D_{n}$, for all $n \geq 1$.

In fact, it is obvious that $F \subset D_{1}$. Suppose that $F \subset D_{n}$. Hence for any $u \in F \subset D_{n}$, by (2.4), we have

$$
\begin{aligned}
& \phi\left(u, y_{n, i}\right)=\phi\left(u, J^{-1}\left(\alpha_{n} J x_{1}+\left(1-\alpha_{n}\right) J z_{n}\right)\right) \\
& \leq \alpha_{n} \phi\left(u, x_{1}\right)+\left(1-\alpha_{n}\right) \phi\left(u, z_{n}\right) \\
& \leq \alpha_{n} \phi\left(u, x_{1}\right)+\left(1-\alpha_{n}\right)\left\{\phi\left(u, x_{n}\right)+v_{n} \zeta\left[\phi\left(u, x_{n}\right)\right]+\mu_{n}\right\} \\
& \leq \alpha_{n} \phi\left(u, x_{1}\right) \\
& +\left(1-\alpha_{n}\right)\left\{\phi\left(u, x_{n}\right)+v_{n} \sup _{p \in F} \zeta\left[\phi\left(p, x_{n}\right)\right]+\mu_{n}\right\} \\
& =\alpha_{n} \phi\left(u, x_{1}\right)+\left(1-\alpha_{n}\right) \phi\left(u, x_{n}\right)+\xi_{n}, \forall i \geq 1 .
\end{aligned}
$$

Therefore we have

$$
\sup _{i \geq 1} \phi\left(u, y_{n, i}\right) \leq \alpha_{n} \phi\left(u, x_{1}\right)+\left(1-\alpha_{n}\right) \phi\left(u, x_{n}\right)+\xi_{n} \text {. }
$$

This shows that $u \in F \subset D_{n+1}$ and so $F \subset D_{n}$. The conclusions are proved.

(III) Now we prove that $\left\{x_{n}\right\}$ converges strongly to some point $p^{*} \in D$.

In fact, since $x_{n}=\Pi_{D_{n}} x_{1}$, from Lemma 2.1(c), we have $\left\langle x_{n}-y, J x_{1}-J x_{n}\right\rangle \geq 0, \quad \forall y \in D_{n}$. Again since $F \subset D_{n}$, we have $\left\langle x_{n}-u, J x_{1}-J x_{n}\right\rangle \geq 0, \forall u \in F$. It follows from Lemma 2.1(b) that for each $u \in F$ and for each $n \geq 1$,

$$
\begin{aligned}
& \phi\left(x_{n}, x_{1}\right)=\phi\left(\Pi_{D_{n}} x_{1}, x_{1}\right) \\
& \leq \phi\left(u, x_{1}\right)-\phi\left(u, x_{n}\right) \leq \phi\left(u, x_{1}\right) .
\end{aligned}
$$

Therefore, $\left\{\phi\left(x_{n}, x_{1}\right)\right\}$ is bounded, and so is $\left\{x_{n}\right\}$. Since $x_{n}=\prod_{D_{n}} x_{1}$ and $x_{n+1}=\Pi_{D_{n+1}} x_{1} \in D_{n+1} \subset D_{n}$, we have $\phi\left(x_{n}, x_{1}\right) \leq \phi\left(x_{n+1}, x_{1}\right)$.

This implies that $\left\{\phi\left(x_{n}, x_{1}\right)\right\}$ is nondecreasing. Hence $\lim _{n \rightarrow \infty} \phi\left(x_{n}, x_{1}\right)$ exists. Since $X$ is reflexive, there exists a subsequence $\left\{x_{n_{i}}\right\} \subset\left\{x_{n}\right\}$ such that $x_{n_{i}} \rightarrow p^{*}$ (some point in $\left.D=D_{1}\right)$. Since $D_{n}$ is closed and convex and $D_{n+1} \subset D_{n}$. This implies that $D_{n}$ is weakly closed and $p^{*} \in D_{n}$ for each $n \geq 1$. In view of

$x_{n_{i}}=\Pi_{D_{n_{i}}} x_{1}$, we have $\phi\left(x_{n_{i}}, x_{1}\right) \leq \phi\left(p^{*}, x_{1}\right), \forall n_{i} \geq 1$. Since the norm $\|\cdot\|$ is weakly lower semi-continuous, we have

$$
\begin{aligned}
& \liminf _{n_{i} \rightarrow \infty} \phi\left(x_{n}, x_{1}\right) \\
& =\liminf _{n_{i} \rightarrow \infty}\left(\left\|x_{n_{i}}\right\|^{2}-2\left\langle x_{n_{i}}, J x_{1}\right\rangle+\left\|x_{1}\right\|^{2}\right) \\
& \geq\left\|p^{*}\right\|^{2}-2\left\langle p^{*}, J x_{1}\right\rangle+\left\|x_{1}\right\|^{2}=\phi\left(p^{*}, x_{1}\right)
\end{aligned}
$$

and so

$$
\begin{aligned}
\phi\left(p^{*}, x_{1}\right) & \leq \liminf _{n_{i} \rightarrow \infty} \phi\left(x_{n}, x_{1}\right) \\
& \leq \limsup _{n_{i} \rightarrow \infty} \phi\left(x_{n}, x_{1}\right)=\phi\left(p^{*}, x_{1}\right) .
\end{aligned}
$$

This shows that $\lim _{n_{i} \rightarrow \infty} \phi\left(x_{n_{i}}, x_{1}\right)=\phi\left(p^{*}, x_{1}\right)$ and we have $\left\|x_{n_{i}}\right\| \rightarrow\left\|p^{*}\right\|$. Since $x_{n_{i}} \rightarrow p^{*}$, by virtue of KadecKlee property of $X$, we obtain that $\lim x_{n_{i}}=p^{*}$. Since $\left\{\phi\left(x_{n}, x_{1}\right)\right\}$ is convergent, this together ${ }^{i}$ with $\lim _{n_{i} \rightarrow \infty} \phi\left(x_{n_{i}}, x_{1}\right)=\phi\left(p^{*}, x_{1}\right)$ shows that $\lim _{n_{i} \rightarrow \infty} \phi\left(x_{n}, x_{1}\right)=\phi\left(p^{*}, x_{1}\right)$. If there exists some subsequence $\left\{x_{n_{j}}\right\} \subset\left\{x_{n}\right\}$ such that $x_{n_{j}} \rightarrow q$, then from Lemma 2.1, we have

$$
\begin{aligned}
\phi\left(p^{*}, q\right) & =\lim _{n_{i}, n_{j} \rightarrow \infty} \phi\left(x_{n_{i}}, x_{n_{j}}\right)=\lim _{n_{i}, n_{j} \rightarrow \infty} \phi\left(x_{n_{i}}, \Pi_{D_{n_{j}}} x_{1}\right) \\
& \leq \lim _{n_{i}, n_{j} \rightarrow \infty}\left[\phi\left(x_{n_{i}}, x_{1}\right)-\phi\left(\Pi_{D_{n_{j}}} x_{1}, x_{1}\right)\right] \\
& =\lim _{n_{i}, n_{j} \rightarrow \infty}\left[\phi\left(x_{n_{i}}, x_{1}\right)-\phi\left(x_{n_{j}}, x_{1}\right)\right] \\
& =\phi\left(p^{*}, x_{1}\right)-\phi\left(p^{*}, x_{1}\right)=0,
\end{aligned}
$$

i.e., $p^{*}=q$ and hence

$$
x_{n} \rightarrow p^{*} .
$$

By the way, from (3.4), it is easy to see that

$$
\xi_{n}=v_{n} \sup _{p \in F} \zeta\left[\phi\left(p, x_{n}\right)\right]+\mu_{n} \rightarrow 0 .
$$

(IV) Now we prove that $p^{*} \in F$.

In fact, since $x_{n+1} \in D_{n+1}$, from (3.1), (3.4) and (3.5), we have

$$
\begin{aligned}
& \sup _{i \geq 1} \phi\left(x_{n+1}, y_{n, i}\right) \\
& \leq \alpha_{n} \phi\left(x_{n+1}, x_{1}\right)+\left(1-\alpha_{n}\right) \phi\left(x_{n+1}, x_{n}\right)+\xi_{n} \rightarrow 0 .
\end{aligned}
$$

Since $x_{n} \rightarrow p^{*}$, it follows from (3.6) and Lemma 2.2 that

$$
y_{n, i} \rightarrow p^{*}(n \rightarrow \infty)
$$

Since $\left\{x_{n}\right\}$ is bounded and $\left\{T_{i}\right\}$ is a countable family of uniformly totally quasi- $\phi$-asymptotically nonexpansive multi-valued mappings, $z_{n} \in T_{i}^{n} x_{n}$ is bounded. In view of $\alpha_{n} \rightarrow 0$, from (3.1), we have

$$
\lim _{n \rightarrow \infty}\left\|J y_{n, i}-J z_{n}\right\|=\lim _{n \rightarrow \infty} \alpha_{n}\left\|J X_{1}-J z_{n}\right\|=0 .
$$

Since $J y_{n, i} \rightarrow J p^{*}$, this implies $J z_{n} \rightarrow J p^{*}$. From Remark 2.1, it yields that

$$
z_{n} \rightarrow p^{*} .
$$

Again since

$$
\left\|z_{n}\right\|-\left\|p^{*}\right\|=\left\|J z_{n}\right\|-\left\|J p^{*}\right\| \leq\left\|J z_{n}-J p^{*}\right\| \rightarrow 0,
$$


this together with (3.9) and the Kadec-Klee-property of $X$ shows that

$$
z_{n} \rightarrow p^{*}
$$

On the other hand, by the assumptions that $T_{i}$ is $L_{i}$-Lipschitz continuous for each $i \geq 1$, we have

$$
\begin{aligned}
& d\left(T_{i} z_{n}, z_{n}\right) \\
\leq & d\left(T_{i} z_{n}, z_{n+1}\right)+\left\|z_{n+1}-x_{n+1}\right\| \\
& +\left\|x_{n+1}-x_{n}\right\|+\left\|x_{n}-z_{n}\right\| \\
\leq & \left(L_{i}+1\right)\left\|x_{n+1}-x_{n}\right\|+\left\|z_{n+1}-x_{n+1}\right\|+\left\|x_{n}-z_{n}\right\| .
\end{aligned}
$$

From (3.12) and $x_{n} \rightarrow p^{*}$, we have that $d\left(T_{i} z_{n}, z_{n}\right) \rightarrow 0$. In view of the closeness of $T_{i}$, it yields that $T_{i}\left(p^{*}\right)=\left\{p^{*}\right\}(\forall i \geq 1)$, which implies that $p^{*} \in F$.

(V) Finally we prove that $p^{*}=\Pi_{F} x_{1}$ and so $x_{n} \rightarrow \Pi_{F} x_{1}$.

Let $w=\Pi_{F} x_{1}$. Since $w \in F \subset D_{n}$, we have $\phi\left(p^{*}, x_{1}\right) \leq \phi\left(w, x_{1}\right)$. This implies that

$$
\phi\left(p^{*}, x_{1}\right)=\lim _{n \rightarrow \infty} \phi\left(x_{n}, x_{1}\right) \leq \phi\left(w, x_{1}\right) .
$$

which yields that $p^{*}=w=\Pi_{F} x_{1}$. Therefore, $x_{n} \rightarrow \Pi_{F} x_{1}$. The proof of Theorem 3.1 is completed.

By Remark 2.4, the following corollaries are obtained.

Corollary 3.1 Let $X$ and $D$ be as in Theorem 3.1, and a countable family of mappings $T_{i}: D \rightarrow D$ $(i=1,2,3, \cdots)$ be a closed and uniformly $L_{i}$-Lipschitz continuous a relatively nonexpansive multi-valued mappings. Let $\left\{\alpha_{n}\right\}$ in $(0,1)$ with $\lim _{n \rightarrow \infty} \alpha_{n}=0$. Let $\left\{x_{n}\right\}$ be the sequence generated by

$$
\left\{\begin{array}{l}
x_{1} \in X \text { is arbitrary; } D_{1}=D \\
y_{n, i}=J^{-1}\left[\alpha_{n} J x_{1}+\left(1-\alpha_{n}\right) J z_{n}\right], \quad z_{n} \in T_{i} x_{n} \\
D_{n+1}=\left\{z \in D_{n}: \sup _{i \geq 1} \phi\left(z, y_{n, i}\right)\right. \\
\left.\quad \leq \alpha_{n} \phi\left(z, x_{1}\right)+\left(1-\alpha_{n}\right) \phi\left(z, x_{n}\right)\right\} \\
x_{n+1}=\prod_{D_{n+1}} x_{1} \quad(n=1,2, \cdots)
\end{array}\right.
$$

where $F\left(T_{i}\right)$ is the set of fixed points of $T_{i}$, and $\Pi_{D_{n+1}}$ is the generalized projection of $X$ onto $D_{n+1}$, If $F:=\bigcap_{i=1}^{\infty} F\left(T_{i}\right) \neq \varnothing$ and $F$ is bounded, then $\left\{x_{n}\right\}$ converges strongly to $\Pi_{F} x_{1}$.

Corollary 3.2 Let $X$ and $D$ be as in Theorem 3.1, and a countable family of mappings $T_{i}: D \rightarrow D$

$(i=1,2,3, \cdots)$ be a closed and uniformly $L_{i}$-Lipschitz continuous quasi-phi-asymptotically nonexpansive multivalued mappings with nonnegative real sequences $\left\{k_{n}\right\} \subset[1,+\infty)$ and $k_{n} \rightarrow 1$ satisfying condition (2.1). Let $\left\{\alpha_{n}\right\}$ be a sequence in $(0,1)$ and satisfy $\lim _{n \rightarrow \infty} \alpha_{n}=0$. If $\left\{x_{n}\right\}$ is the sequence generated by

$$
\left\{\begin{aligned}
x_{1} \in X & \text { is arbitrary; } \quad D_{1}=D \\
y_{n, i}=J^{-1}\left[\alpha_{n} J x_{1}+\left(1-\alpha_{n}\right) J z_{n}\right], \quad z_{n} \in T_{i}^{n} x_{n} & \\
D_{n+1}= & \left\{z \in D_{n}: \sup _{i \geq 1} \phi\left(z, y_{n, i}\right)\right. \\
& \left.\quad \leq \alpha_{n} \phi\left(z, x_{1}\right)+\left(1-\alpha_{n}\right) \phi\left(z, x_{n}\right)+\xi_{n}\right\} \\
x_{n+1}= & \Pi_{D_{n+1}} x_{1} \quad(n=1,2, \cdots)
\end{aligned}\right.
$$

where $F\left(T_{i}\right)$ is the set of fixed points of $T_{i}$, and $\Pi_{D_{n+1}}$ is the generalized projection of $X$ onto $D_{n+1}$, and $\xi_{n}=\left(k_{n}-1\right) \sup _{p \in F} \phi\left(p, x_{n}\right)$.

If $F:=\bigcap_{i=1}^{\infty} F\left(T_{i}\right) \neq \varnothing$ and $F$ is bounded, then $\left\{x_{n}\right\}$ converges strongly to $\Pi_{F} X_{1}$.

\section{Application}

We utilize Corollary 3.2 to study a modified Halpern iterative algorithm for a system of equilibrium problems.

Theorem 4.1 Let $D, X$ and $\left\{\alpha_{n}\right\}$ be the same as in Theorem 3.1. Let $f: D \times D \rightarrow R$ be a bifunction satisfying conditions (A1)-(A4) as given in Example 2.6.

Let $T_{r}: X \rightarrow D$ be a mapping defined by (2.5), i.e.,

$$
\begin{aligned}
& T_{r}(x) \\
& =\left\{x \in D, f(z, y)+\frac{1}{r}\langle y-z, J z-J x\rangle \geq 0, \forall y \in D\right\},
\end{aligned}
$$$$
\forall x \in X \text {. }
$$

Let $\left\{x_{n}\right\}$ be the sequence generated by

$$
\left\{\begin{array}{c}
x_{1} \in X \text { is arbitrary; } D_{1}=D \\
f\left(u_{n}, y\right)+1 r\left\langle y-u_{n}, J u_{n}-J x_{n}\right\rangle \geq 0, \\
\quad \forall y \in D, r>0, u_{n} \in T_{r} x_{n} \\
y_{n}=J^{-1}\left[\alpha_{n} J x_{1}+\left(1-\alpha_{n}\right) J u_{n}\right] \\
D_{n+1}=\left\{z \in D_{n}: \phi\left(z, y_{n}\right)\right. \\
\left.\leq \alpha_{n} \phi\left(z, x_{1}\right)+\left(1-\alpha_{n}\right) \phi\left(z, x_{n}\right)\right\} \\
x_{n+1}=\prod_{D_{n+1}} x_{1}(n=1,2, \cdots) .
\end{array}\right.
$$

If $F\left(T_{r}\right) \neq \Phi$, then $\left\{x_{n}\right\}$ converges strongly to $\prod_{F(T)} X_{1}$ which is a common solution of the system of equilibrium problems for $f$.

Proof. In Example 2.6, we have pointed out that $u_{n}=T_{r}\left(x_{n}\right), F\left(T_{r}\right)=E P(f)$ and $T_{r}$ is a closed quasi- $\phi$-nonexpansive mapping. Hence (4.1) can be rewritten as follows:

$$
\left\{\begin{array}{l}
x_{1} \in X \text { is arbitrary; } D_{1}=D \\
y_{n}=J^{-1}\left[\alpha_{n} J x_{1}+\left(1-\alpha_{n}\right) J u_{n}\right], \quad u_{n} \in T_{r} x_{n} \\
D_{n+1}=\left\{z \in D_{n}: \phi\left(z, y_{n}\right)\right. \\
\left.\quad \leq \alpha_{n} \phi\left(z, x_{1}\right)+\left(1-\alpha_{n}\right) \phi\left(z, x_{n}\right)\right\} \\
x_{n+1}=\prod_{D_{n+1}} x_{1}(n=1,2, \cdots) .
\end{array}\right.
$$


Therefore the conclusion of Theorem 4.6 can be obtained from Corollary 3.2.

\section{REFERENCES}

[1] I. Cioranescu, "Geometry of Banach Spaces, Duality Mappings and Nonlinear Problems," Kluwer Academic, Dordrecht, 1990. doi:10.1007/978-94-009-2121-4

[2] W. R. Mann, "Mean Value Methods in Iteration," Proceedings of the American Mathematical Society, Vol. 4, No. 3, 1953, pp. 506-510. doi:10.1090/S0002-9939-1953-0054846-3

[3] A. Genel and J. Lindenstrauss, "An Example Concerning Fixed Points," Israel Journal of Mathematics, Vol. 22, No. 1, 1975, pp. 81-86.

[4] B. Halpren, "Fixed Points of Nonexpansive Maps," Bulletin of the American Mathematical Society, Vol. 73, 1967, pp. 957-961.

doi:10.1090/S0002-9904-1967-11864-0

[5] K. Nakajo and W. Takahashi, "Strong Convergence Theorems for Nonexpansive Mappings and Nonexpansive Semigroups," Journal of Mathematical Analysis and Applications, Vol. 279, No. 2, 2003, pp. 372-379. doi:10.1016/S0022-247X(02)00458-4

[6] K. Aoyama and Y. Kimura, "Strong Convergence Theorems for Strongly Nonexpansive Sequences," Applied Mathematics and Computation, Vol. 217, No.19, 2011, pp. 7537-7545. doi:10.1016/j.amc.2011.01.092

[7] S. S. Chang, H. W. J. Lee, C. K. Chan and W. B. Zhang, "A Modified Halpern-Type Iteration Algorithm for Totally Quasi- $\phi$-Asymptotically Nonexpansive Mappings with Applications," Applied Mathematics and Computation, Vol. 218, No. 11, 2012, pp. 6489-6497. doi:10.1016/j.amc.2011.12.019

[8] S. S. Chang, L. Yang and J. A. Liu, "Strong Convergence Theorem for Nonexpansive Semi-Groups in Banach Space," Applied Mathematics and Mechanics, Vol. 28, No. 10, 2007, pp. 1287-1297. doi:10.1007/s10483-007-1002-x

[9] C. E. Chidume and E. U. Ofoedu, "Approximation of Common Fixed Points for Finite Families of Total Asymptotically Nonexpansive Mappings," Journal of Mathematical Analysis and Applications, Vol. 333, No. 1, 2007, pp. 128-141. doi:10.1016/j.jmaa.2006.09.023

[10] S. Matsushita and W. Takahashi, "Weak and Strong Con- vergence Theorems for Relatively Nonexpansive Mappings in a Banach Space," Fixed Point Theory and Applications, Vol. 2004, 2004, pp. 37-47. doi:10.1155/S1687182004310089

[11] S. Matsushita and W. Takahashi, "An Iterative Algorithm for Relatively Nonexpansive Mappings by Hybrid Method and Applications," Proceedings of the 3rd International Conference on Nonlinear Analysis and Convex Analysis, Tokyo, 2004, pp. 305-313.

[12] S. Matsushita and W. Takahashi, "A Strong Convergence Theorem for Relatively Nonexpansive Mappings in a Banach Space," The Journal of Approximation Theory, Vol. 134, No. 2, 2005, pp. 257-266. doi:10.1016/j.jat.2005.02.007

[13] X. L. Qin, Y. J. Cho, S. M. Kang and H. Y. Zhou, "Convergence of a Modified Halpern-Type Iterative Algorithm for Quasi- $\phi$-Nonexpansive Mappings," Applied Mathematics Letters, Vol. 22, No. 7, 2009, pp. 1051-1055. doi:10.1016/j.aml.2009.01.015

[14] Y. Song, "New Strong Convergence Theorems for Nonexpansive Nonself-Mappings without Boundary Conditions," Computers and Mathematics with Applications, Vol. 56, No. 6, 2008, pp. 1473-1478. doi:10.1016/j.camwa.2008.03.004

[15] Z. M. Wang, Y. F. Su, D. X. Wang and Y. C. Dong, "A Modified Halpern-Type Iteration Algorithm for a Family of Hemi-Relative Nonexpansive Mappings and Systems of Equilibrium Problems in Banach Spaces," The Journal of Computational and Applied Mathematics, Vol. 235, No. 8, 2011, pp. 2364-2371. doi:10.1016/i.cam.2010.10.036

[16] Y. I. Alber, "Metric and Generalized Projection Operators in Banach Spaces: Properties and Applications," In: A. G. Kartosator, Ed., Theory and Applications of Nonlinear Operators of Accretive and Monotone Type, Dekker, New York, 1996, pp. 15-50.

[17] S. S. Chang, C. K. Chan and H. W. J. Lee, "Modified Block Iterative Algorithm for Quasi- $\phi$-Asymptotically Nonexpansive Mappings and Equilibrium Problem in Banach Spaces," Applied Mathematics and Computation, Vol. 217, No. 18, 2011, pp. 7520-7530. doi:10.1016/j.amc.2011.02.060

[18] E. Blum and W. Oettli, "From Optimization and Variational Inequalities to Equilibrium Problems," Mathematical Studies, Vol. 63, No. 1/4, 1994, pp. 123-145. 